\title{
Go forth and replicate
}

\author{
The motto of the Royal Society, "nullius in verba", enjoins scientists to 'take nobody's word for it'. Its call to trust \\ nothing unless it can be replicated is no less relevant now than it was 360 years ago.
}

$\mathrm{S}$ cientific understanding is always provisional. Any model or theory, however well supported by evidence, can be falsified by a single result. Scientists are also fallible; the results of experiments cannot always be reproduced due to errors, unappreciated complications or just that even the most improbable things can and will happen sometimes. This is why we publish corrections to papers on a fairly regular basis and retractions, hopefully less frequently. There are also Matters Arising (such as the recent Nutrient scarcity cannot cause mast seeding from David Kelly ${ }^{1}$ ), usually accompanied by a reply from the original study's authors. This is all a normal part of the process of scientific discovery.

Despite this, there is much talk of a 'reproducibility crisis'. The pressure to publish and the bias towards publication of positive results has led, so it is said, to increasing numbers of studies that are untrustworthy or just plain wrong. Confidence in science is only maintained by swiftly identifying and correcting such studies; preferably in the same venue as the original publication. It was therefore intriguing to see a recent 'failure-to-reproduce' paper ${ }^{2}$ appearing in a journal not only different from its antecedent ${ }^{3}$, but one generally regarded as more prestigious. The reason is not too hard to understand, as these studies concern the vexed question of plant intelligence.

Plants indulge in a huge variety of complex behaviours. They sense the world around them and change their growth, metabolism and physiology in response. Whether this is considered 'intelligent' has a lot to do with your definition of the term. Indeed, Daniel Chamovitz ${ }^{4}$ has argued in our pages that this question is an unhelpful distraction from studying these important phenomena. However, the paper by Gagliano et al. published in Scientific Reports in 2016 concerns the well-defined behaviour known as 'associative learning', the best-known example being the work on the dogs of Ivan Pavlov, for which he received the Nobel Prize for Physiology or Medicine in 1904.

Some attempts were made in the 1960 s to determine whether plants could be similarly conditioned using Mimosa pudica, the 'sensitive plant' or 'touch-me-not', which curls up its leaves as a defence mechanism when disturbed. These conditioning experiments involved electric shocks, changes in light regimes and dropping of the plants as stimuli. The results were equivocal at best, and, with hindsight, the methodologies seem riddled with serious limitations.

The best known of those now historical studies were published in Worm Runner's Digest and latterly the Journal of Biological Psychology, two publications established by the psychologist James V. McConnell. In 1959, McConnell presented work on the "apparent retention of a conditioned response following total regeneration in the planarian" at an American Psychological Association meeting. This provided data suggesting that separate pieces of trained flatworms, after being allowed to regenerate, retained the training given to the original worm. As a result of press reports, McConnell received so many enquiries from school students who wanted to use the worms for science projects that he wrote a guide describing in detail how to repeat the experiments. As a joke, he labelled it Worm Runner's Digest Vol. 1. No 1 , the consequence of which was that McConnell started receiving submissions to this 'new' journal.

Despite containing legitimate scientific studies, Worm Runner's Digest never took itself particularly seriously, including poems, cartoons, spoofs and jokes in its pages, and was, in McConnell's words, "one of the first scientific journals that knowingly published satire". By 1964, there were so many complaints from readers unable to tell the spoofs from the sincere that the two were separated, with the jokes consigned to the back of each issue and printed upside down. In 1967, the two halves became permanently separated, the front becoming the Journal of Biological Psychology.

Despite their problems, the M. pudica experiments have not been forgotten. In 2016, Monica Gagliano of the University of Western Australia used the paradigm of habituating $M$. pudica to a series of drops to propose that they 'ignored' repeated and irrelevant stimuli more in conditions of poor illumination where the costs of unnecessarily closing their leaves were higher ${ }^{5}$.

Such a response falls short of demonstrating associative learning, and so Gagliano turned her attention to pea plants, Pisum sativum. Single plants were grown in Y-mazes with lights and fans attached. When the light in one arm is turned on, the plant grows towards it. Fans were turned on an hour before the light, blowing air down the same or opposite arms of the maze. Over time, the direction of growth of the peas was affected by the position of the fan alone, as though the plants had learnt to predict where the light would later appear.

Unsurprisingly, what looked like a clear demonstration that individual plants could both learn and remember received considerable attention. A profile of Gagliano appeared in the New York Times ${ }^{6}$ and the study has been cited at least 45 times. But time moves on, and now Kasey Markel of University of California, Davis, is reporting that repeating the protocol using more plants, additional control conditions and more explicit blinding during scoring of the results does not produce the same marked affects and fails to support the earlier conclusions.

Markel's paper demonstrates a crucial problem for replication studies: the difficulty in ensuring that the experiments are performed in exactly the same way. For example, Markel could not obtain exactly the same variety of pea, and is uncertain as to the source of fans and light-emitting diode lights. It seems unlikely that these factors would have changed the outcome of the experiments substantially, but they might have.

The only test that matters in science is the test of time. Markel's doubts over Gagliano's results may themselves be overturned next month, or next year, or next decade. Whatever the topic, we must remain open to not only the dramatic result but also the reasonable doubt about its capacity for replication.

Published online: 11 August 2020 https://doi.org/10.1038/s41477-020-00759-z

References

1. Kelly, D. Nat. Plants 6, 760-762 (2020).

2. Markel, K. eLife 9, e57614 (2020).

3. Gagliano, M. et al. Sci. Rep. 6, 38427 (2016).

4. Chamovitz, D. A. Nat. Plants 4, 622-623 (2018).

5. Gagliano, M. et al. Oecologia 175, 63-72 (2014).

6. Shechet, E. Do Plants Have Something to Say? New York Times https://www.nytimes.com/2019/08/26/style/can-plants-talk. html (2019). 